\title{
Analysis on Psychological Crisis Intervention of Young College Students
}

\author{
Qi Wang
}

Zhengzhou Vocational University of Information and Technology, Zhengzhou, 450046, China

\author{
Keywords: Colleges and universities, Young Students, Psychological crisis intervention
}

\begin{abstract}
With the employment situation of college students become severer and severer in recent years, college students suffered from various psychological crises in employment as lack of selfadjustment ability and consequently affected their normal employment and future life. However, the colleges in China lack of young students' psychological crisis intervention mechanism. On that account, strengthening the study on problems and countermeasures of young students' psychological crisis intervention is of great urgency. This thesis analyzed the concept and its causes of young students' psychological crisis intervention, discussed the principle of young students' psychological crisis intervention and proposed corresponding countermeasures.
\end{abstract}

\section{Introduction}

In the current broad environment of global economic integration, the material life and think mode of modern people changed, and psychological activities of the young college students in colleges are more complicated and changeable, resulting in intense psychological conflicts and contradictions between ideal and reality, emotion and reason, impulsion and repression, and in turn cause psychological crises. It is thus clear that the young college students' psychological crisis intervention has become a pressing issue to be addressed urgently.

\section{The related meanings of psychological crisis intervention of young college students}

Crisis mainly has two implications: one refers to the unexpected emergency, such as earthquake, floods, fires, terrorist attacks, and so on. Another refers to people in the state of emergency condition. Once an individual encounters significant problem or change which is difficult for the individual to resolve, thus the individual will completely change the balance, lead to continuous accumulation of a sense of inner tension and cause the individual at a loss even confusion, and then enter into an unbalanced state, which is a crisis state. This means that the balance and stability is destroyed, which led to confusion and disturbance. Any slight change in the college students' growing process may produce changes in lifestyles, once they can not adjust in time, it will easily result in psychological crisis. Psychological crisis either means that people's psychological state is in a serious imbalance state, leading to intense psychological conflicts and contradictions that can not be solved, or means the spirit of a person facing a collapse or mental disorders, or refers to the emergence of mental disorders.

Psychological crisis intervention means that under the guidance of psychology, to assist the individual or groups who suffered from psychological crisis for short term. It aims to timely provide support and help for people who is experiencing personal crisis, plight or suffered from frustration as well as the one who is prone to suffering from crisis and help them to restore psychological balance. Psychological crisis intervention is not equal to common psychological counseling and therapy, instead, the major features of psychological crisis intervention are timely and rapid, and the effective action is the key to achieve success. Colleges and universities should actively establish and improve the overall psychological crisis intervention mechanism to effectively prevent crises to eliminate the issue in the initial state. 


\section{The causes of the young college students' psychological crisis}

Firstly, it is caused by the difficulties in study. Most students are top students in their class before they enrolled in the college, and their parents and themselves place high expectations on them, however, after entered the college, the competition is fiercer than before, and the studying mode are significantly different from before, thus the learning contents become deeper and wider, and put more requirements on independent arrangement in learning process and arrangement on learning time. As a result, some students with relatively poor adaptive capacity fail to handle these changes, and suffer from a disastrous decline in study performance, cause them great frustration and then result in psychological crisis. Secondly, it is caused by the difficulties in life. At present, the annual expenditure of college students exceeds 10 thousand Yuan, which is affordable for the families of developed regions, but for the students from poverty-stricken areas is a heavy burden. Hereby, some poverty students tend to shoulder a heavy burden, and their behaviors tend to be rather radical and prone to the extreme. Thirdly, it is caused by the difficulties in communication. Most of the college students are only child who has been completely relying on their family since childhood and think they deserve to be taken care of, and they don't know how to care for others and without sense of gratitude. After entered the college, they have to live together and how to get along with classmates and roommates become an urgent problem. If they fail to deal with the problem and after long-term accumulation, psychological crisis may be caused. Fourthly, it is caused by the emotional trouble. It is quite common for male and female students in colleges fall in love, but as they lack of social experience, they are more pure in thought and their attitude towards love is too idealistic, thus they are prone to extreme, and break up with each other once meet a conflict. Under this situation, how to properly adjust the psychology of college students who are crossed in love is very important.

\section{The principle of psychological crisis intervention of the young college students}

Firstly, the principle of "Preventive Education First”. At present, vast majority of colleges are still in the stage of remedial intervention, and most of them manage to remedy after the crisis has produced adverse consequences. The crisis intervention is just as firefighters which is very passive, and the consequences are often only slightly eased the psychological imbalance of the college students, it is thus clear that restoring their normal mental function is necessary. As for the college students with suicidal tendencies, omen and rules can be found before that happen. Colleges should provide psychological education with the students since they are enrolled in the college and screen out the students with crises to carry out crisis intervention. At the same time, the colleges should note that the crises prevention can not only improve the college students' psychological health status and coping capacity, but also can improve their ability to help others, thereby effectively increase the capacity to solve all kinds of psychological crises.

Secondly, the principle of timeliness. Psychological crisis is a kind of emergency, thus timeliness is the key factor in the process of psychological crisis intervention and attempts are not allowed to make. On that account, psychological crisis intervener should make rapid response and treatment on the emerged and continuously changed issues in the process of crisis, and timely discover report, intervene and refer, as well as continue to keep track of the students' ideological trends, so as to provide appropriate assistance to them according to their own needs. Otherwise, the ideal time to intervene the crisis will be missed, and in consequence, the crisis will develop into uncontrolled state.

Thirdly, the principle of value neutrality. Once a young college student suffered from a psychological crisis due to the factors of academic frustration, be crossed in love, interpersonal conflict, etc., the causes have a very close relationship with their personal values. In the course of implementing psychological crisis intervention on young college students, the counseling staff should be based on the principle of value neutrality. The value neutrality here mainly refers to that the staff should temporarily set aside his value, do not evaluate the visitor's personal value, instead, he should concentrate on the understanding and sympathy for the visitor. The Counseling staff should accept the visitor's value unconditionally, although their values are mutually opposite, the staff can 
not arbitrarily criticize the visitor or optionally evaluate the visitor. In other words, the college educators should aloof to the conflict of values between the two sides and to create a harmonious and relaxing atmosphere with a neutral attitude.

\section{The countermeasures for psychological crisis intervention of the young college studentsguide the values of the young college students}

Value is an important foundation for people's psychological health, and the occurrence of psychological crisis is generally has very close ties with people's values. A large number of facts proved that the conflict of values is an important factor causing psychological crisis on college students. Therefore, strengthening the psychological crisis prevention has become a priority in the process of shaping a healthy personality. It is necessary to guide students to form a new value system that coordinated with their cultural environment, so as to allow them to learn to assess and control their own actions and thoughts as they are facing with conflicts in their life. In order to solve the young college students' psychological crisis, the educational value of college talents need to be continuously updated, the educational methods should be changed and the related contents of psychological crisis intervention should be richened. For instance, we absolutely can improve the educational value through exploring the meaning of existence, and change the education and teaching modes through creating a harmonious campus environment.

To perfect the young college students' psychological crisis prevention system

As an essential basic step for psychological crisis intervention, psychological crisis prevention should be sound and perfect. Establishing the psychological crisis prevention system of young college students in accordance with the following points: firstly, make full use of various forms, such as electives, seminars, counseling psychology hotlines and websites, etc., to guide college students to timely and effectively master the common knowledge of psychological crisis, guide them to establish a model of psychological awareness and help them continue to improve the ability to respond to crises. Secondly, in the team construction, it should strengthen the psychological health education and basic training in psychological intervention capability of the contingent of cadres, counselors, teachers and student management staff, especially the full-time student management team, to help them to possess psychological crisis intervention awareness and capacity. Thirdly, strive to build a warm and harmonious campus cultural environment. Harmonious campus culture is one of the major conditions for college students to develop healthily. Through the implementation of a variety of campus culture construction in colleges to better meet their physical and psychological needs, and provide a good platform for them to show their talents, express their passion from heart, effectively enhance the competitive strength, and get stronger confidence.

To perfect the warning and monitoring system of young college students' psychological crisis

Psychological crisis warning has become an essential part of psychological crisis intervene. Psychological crisis warning system should possess subsystems of psychological crisis prevention, intervene, etc., to fully exert the functions of information transmission, screening, integration, reporting, and so on. Firstly, it should establish "five levels" warning and monitoring system in colleges. Here, the "five levels" refers to the five levels' system in college students' dormitory, classroom, colleges and departments, college psychological counseling centers and the college. Monitoring the students' dormitory can carefully observe the actions and psychological trends of the students with crisis. Monitoring the classrooms can observe the class leaders, and truly understand the psychological changes of the students in the class. Monitoring the colleges and departments can establish a bridge between the students and the corresponding colleges and departments, so as to provide various help for them. Monitoring the psychological counseling centers can provide professional psychological counseling service to the students groups. Monitoring the college can provide adequate coordination and guidance for students in psychological crisis intervention. Thirdly, establish psychological health screening system. The colleges should implement annual psychological health screening on college students, and find out the students with psychological 
crisis tendency according to the results, set his psychological crisis intervention warning database, as well as guide and help the students truly have psychological disorder after regular checking and observation.

\section{To form young college students' psychological crisis intervention system}

Colleges should strengthen the resources integration to implement intervention on the students' high risks, and actively build a comprehensive psychological crisis intervention system. Firstly, pay attention to the students groups with high incidence of psychological crisis, implement intervention and control on them. Emphasize to help the students who come from poor family, with poor learning conditions, suffer from interpersonal obstacles and love trouble, and effectively help them to solve practical problems. Secondly, integrate various psychological intervention resources on and off campus, fully exert united force to implement college students' psychological crisis intervention. Perfect the psychological crisis intervention institutions, timely provide effective help for students with psychological crisis tendency. Thirdly, implement social and family psychological crisis prevention system. Actively integrate various social forces of the family member, friends and classmates of the students with high psychological crisis risk, as well as social psychosocial service personnel, working staff in media business, etc, to actively involve in the crisis intervention of the students. Give them sincere love, comfort and help to let them form nostalgia to their family, school, relatives, friends and life, effectively enhance their expectation value for the future new life, so that re-generate new hope for their future, and actively cooperate with treatment, thereby ensuring to overcome the psychological crisis .

\section{Conclusion}

It is obvious that young college students' psychological crisis intervention is important issue for teaching staff in colleges, which is sure to attract more attention of the whole society and should explore a truly efficient, effective and feasible intervention measures through practical research. We should not only strengthen the prevention to avoid young college students' psychological crisis to utmost extent, but also adopt effective countermeasures to intervene once the crisis break out thereby to promote the harmonious construction of campus.

\section{References}

[1] Zhuo Gaosheng: Study on Occurrence Origin of Psychological Crisis for College Students and Its Interfering, Study on Ideological and Political Work, 2009 (12)

[2]Zhang Haitao, Su Ling: Analysis on the Establishing of College Students' Psychological Crisis Intervention, School Party Building and Ideological Education, 2010 (5)

[3]Zhao Shengyu: Case Analysis on College Students’ Psychological Crisis Intervention, China New Technology and New Products, 2010 (8)

[4]Lu Qin: Analysis on the Establishing of College Students’ Psychological Crisis Prevention and Intervention System, China Youth Research 2010 (9)

[5]Jia Xin, Li Yubo: Case Analysis and Thoughts on College Students' Psychological Crisis Intervention, New West, 2011 (15) 